ENSEÑANZAS DE LA

METODOLOGÍA 



\title{
Investigar en cárceles. Apuntes para futuros cientificos sociales
}

Research in prisons. Notes for future social scientists

\author{
Antonio Viedma Rojas \\ Universidad Nacional de Educación a Distancia \\ aviedma@poli.uned.es (ESPAÑA) \\ Consuelo del VAl Cid \\ Universidad Nacional de Educación a Distancia \\ cval@poli.uned.es (ESPAÑA) \\ Javier Callejo Gallego \\ Universidad Nacional de Educación a Distancia \\ mcallejo@poli.uned.es(ESPAÑA)
}

Recibido: 11.062018

Aceptado: 17.12.2018

\section{RESUMEN}

Este documento es el resultado de una reflexión sobre las posibilidades y dificultades de la observación social empírica en la cárcel, desde la preocupación por la enseñanza de la metodología sociológica aplicada a las titulaciones universitarias de criminología. Lo aquí expuesto se apoya en la experiencia práctica que los autores han acumulado a través de múltiples estudios realizados en España y en distintos países de Iberoamérica. Una primera versión del texto fue creada para ser utilizada como apuntes prácticos de investigación para estudiantes de criminología que comenzaban su formación y no tenían por tanto experiencia profesional en este campo de estudio. A esa versión se le han introducido las adaptaciones oportunas para que pueda ser utilizado de manera más general por investigadores y estudiantes de ciencias sociales que necesiten una guía básica de diseño y ejecución de proyectos de investigación social en cárceles. El texto tiene una orientación didáctica, es una propuesta que ofrece claves para mejorar la en-señanza y aplicación de la metodología de investigación en los sistemas peniten-ciarios. 


\title{
PALABRAS CLAVE
}

Cárceles, institución total, criminología social, investigación social, metodología.

\begin{abstract}
This article is the result of a reflection on the possibilities and difficulties of empirical social observation in prison, from the perspective of sociological methodology teaching applied to university degrees in criminology. What is here exposed is based on the practical experience accumulated by the authors via mul-tiple studies carried out in Spain and in different Ibero-American countries. A first draft of this text was created with the purpose of being used as practical research notes for students of criminology who began their training and had no profession-al experience in this field of study. This version has been adapted, so that it may be used generally by researchers and students of social sciences who need a basic guide to the design and execution of social research projects in prisons. The paper assumes a didactic orientation; it is a proposal that offers guidelines to improve the teaching and application of the research methodology in the penitentiary systems.
\end{abstract}

\section{KEY WORDS}

Prison, total institutions, social criminology, social research, methodology.

\section{INTRODUCCIÓN}

Este documento es el resultado de una reflexión sobre la observación social empírica en la cárcel. Lo aquí expuesto se apoya en la experiencia práctica que los autores han acumulado a través de múltiples estudios en este campo en España y en distintos países de Iberoamérica. Una primera versión del texto fue creada para ser utilizada como apuntes prácticos de investigación para estudiantes de criminología que comenzaban su formación y no tenían por tanto experiencia profesional en este campo de estudio. A esa versión se le han incorporado las adaptaciones oportunas para que pueda ser utilizado de manera más general por investigadores y estudiantes de ciencias sociales que necesiten una guía básica de diseño y ejecución de proyectos de investigación social en cárceles. El texto tiene por tanto una primera orientación didáctica, es una propuesta que ofrece claves para mejorar la enseñanza y aplicación de la metodología de investigación en los sistemas penitenciarios de España. En segundo lugar, es una reflexión sobre las posibilidades y limitaciones para llevar a cabo tal tipo de investigación. 


\section{2. ¿POR QUÉ LA CÁRCEL?}

La inmersión en un campo de estudio criminógeno es una situación compleja e, incluso, peligrosa que debe ser evaluada con detenimiento por el investigador. Compleja puesto que, como define Luhmann (1996:190 y ss.), se presentan distintas alternativas de actuación. Peligrosa porque el observador entra en contacto con humanos. En este caso, además, tales humanos conviven en una institución total que genera autopoiesis en torno al castigo y la transformación de las personas que custodia. El conflicto constante ordena las relaciones. Sin duda alguna, el investigador se enfrenta a una situación difícil de gestionar. ¿Cómo hacerlo? De hecho, las relativamente particulares circunstancias de este campo de observación empírica, ha servido de fuente reflexiva sobre los aspectos latentes -en cuanto no siempre manifiestos- en el proceso de observación empírica en general.

Los estudios del delito han utilizado a lo largo de los años todo tipo de técnicas de investigación y se han llevado a cabo en múltiples escenarios, tanto públicos como privados. Algunos de los primeros trabajos clásicos son ejemplos interesantes. Las autobiografías fueron utilizadas por Saw y Burguer (1930) en su estudio sobre Jack Roller, un joven delincuente cuya historia sirvió para crear uno de los primeros arquetipos delictivos. Las etnografías como la de Whyte (1943) en los barrios marginales de Chicago sirvieron para explicar la vida y la cultura de una zona de inmigración y conflicto. La relación entre pobreza y delincuencia fue puesta bajo el foco investigador y ha sido un tema de debate siempre presente. Las historias de vida como la elaborada por Donald Sutherland (1946) para crear la tipología del ladrón profesional sirvió para explicar que el delito también podía ser considerado por quienes lo ejercían como una actividad "normal". Su estrategia de crear una tipología a partir de historias de vida y entrevistas se ha utilizado desde entonces. La observación micro-social es otro diseño ampliamente utilizado. Es la aportación del interaccionismo simbólico. Uno de sus mejores ejemplos es el realizado por Howard Becker en Outsiders (1963) un trabajo clásico que explica la construcción de la cultura desviada y el etiquetamiento de los fumadores de marihuana. Desde un enfoque estructural, el estudio de medios de comunicación y poder político realizado por Stanley Cohen (1972), en el que nos explica la forma en que se construye el "pánico moral" y la amenaza de grupos etiquetados como desviados y peligrosos socialmente, es otra estrategia de diseño metodológico muchas veces repetida. También se han realizado experimentos sobre dinámicas de interacción en prisiones simuladas (Haney, Banks y Zimbado 1973) o estudios longitudinales de las trayectorias delictivas de cohortes de delincuentes de Laub y Sampson (2006) utilizando los juzgados, los centros de menores y sus hogares. En definitiva, el estudio del delito se ha apropiado de todo el espectro de técnicas y espacios de investigación posibles. ¿Cómo lo ha hecho en las cárcel?

La elección de la cárcel como campo de observación ha sido recurrente desde que la investigación científica comenzó a dar sus primeros pasos. En el siglo XIX, los primeros criminólogos positivistas usaron los cuerpos de los presos 
como material de estudio (Wahidin y Moore 2011: 290 y ss). Los trabajos del médico Lombroso basados en fotografías de personas ejecutadas en la cárcel, sus autopsias o los estudios de los cuerpos tatuados sirvieron para plantear una perspectiva que desgraciadamente ha sido y es recurrente, aunque actualmente se matice, sobre la relación entre criminalidad y la constitución física o biológica de las personas ${ }^{1}$. Posteriormente, esta idea sirvió para legitimar los trabajos que pretendieron justificar el concepto de raza y la eugenesia. Los experimentos realizados durante la II Guerra Mundial en todo tipo de centros de detención puso de manifiesto la necesidad de salvaguardar los derechos de las personas detenidas. La Declaración Universal de los Derechos Humanos (1948) ${ }^{2}$ y el principio legal del consentimiento informado supuso la persecución social y legal de estas conductas y la introducción de una perspectiva ética de investigación en contextos de detención. Aún seguimos desarrollándola y queda mucho por hacer.

Los trabajos clásicos de Sykes (1958) sobre la subcultura carcelaria, Cohen y Taylor (1972) sobre los efectos psicológicos del encarcelamiento, Goffman (1972) sobre la vida en una "institución total" y la genealogía de Foucault (1975) son un buen punto de partida para comenzar a comprender las posibilidades que ofrece la cárcel como espacio de estudio. Sin embargo, los trabajos más recientes e interesantes en este campo se centran en los efectos psicosociales del encarcelamiento (Liebling 1999; Liebling y Maruna edt. 2005), las implicaciones que la sociodiversidad tiene en la ejecución penal (Crewe y Bennett 2012), el papel de la familia durante el cumplimiento de la detención (Codd 2008) o las posibilidades que los efectos de la cárcel tienen sobre los procesos de reintegración social y abandono del delito tras la salida (Maruna y Immarigeon edit. 2004). Así mismo, por lo que se refiere al diseño, puede ser un buen punto de partida para aquellos que consideran el uso de la perspectiva cualitativa de investigación la lectura del Volumen 20, 4 de 2014 de la revista Qualitative Inquiry sobre la investigación en la cárcel de un modo diferente editado por Yvonne Jewkes (2014)

En general y más allá de los específicos objetivos de conocimiento de cada estudio concreto, los motivos que justifican la decisión de investigar en la cárcel tienen que ver con: la relativa seguridad que ofrece al investigador un espacio criminógeno controlado, el acceso a registros de la actividad delictiva, las posibilidades de observar a lo largo del tiempo cambios en las trayectorias vitales de los presos o la disponibilidad de un banco de informantes con tiempo para hablar $\mathrm{y}$, por lo tanto, de ser observados.

Investigar en la cárcel implica, sobre todo, trabajar con sujetos que se encuentran parcialmente aislados de un entorno social elegido ${ }^{3}$, están a cargo de una "institución total" y, por tanto, en una situación de dependencia muy comprometida. La influencia que puede ejercer el sistema para que los encar-

${ }^{1}$ Una búsqueda mínima en Internet permite llegar con facilidad a las imágenes de las tipologías de delincuentes según su aspecto físico que Lombroso creó.

${ }^{2}$ La Declaración Universal de Derechos Humanos está accesible en: http://www.un.org/es/ universal-declaration-human-rights/

${ }^{3}$ En sentido estricto, no puede decirse que los internos estén aislados de la sociedad; aun cuando en buena medida sea lo que la sociedad pretenda. 
celados participen en las investigaciones no suele ser explícita y puede pasar desapercibida para el investigador. La particularidad aquí es que sospechamos de la existencia de tales influencias; mientras que pocas veces nos preguntamos el por qué de los informantes, las razones para constituirse en informantes. Por ejemplo, el informante aparece como punto de partida para estudiar la institución y no como un efecto de la propia institución. Sin embargo, participar o colaborar con la investigación puede ser para estas personas un modo de mostrar adhesión a la acción de la institución o una manifestación de su rebeldía hacia esta. La cooperación en este contexto es siempre interesada y es por eso que se deben considerar las posibles mediaciones de ese interés en todo momento. Hay que cuestionarse e intentar conocer las respuestas a las siguientes preguntas: ¿por qué participan? ¿qué esperan de nosotros? ¿qué mensaje mandan a la institución con su participación? o ¿cómo comprenden nuestro papel? Pero, también es necesario averiguar ¿por qué no participan los potenciales informantes a quien se les ha solicitado, dejando a un lado a quienes ni siquiera han sido pre-seleccionados para participar? Los informes de contactación ocupan en este tipo de investigación un lugar fundamental.

Del mismo modo, es ineludible tener en cuenta los efectos que el contexto de encierro ejerce sobre las personas que cumplen condena. El tiempo y el espacio social de la cárcel atienden a una organización peculiar (Scarce 2002; Viedma 2013). Ambas dimensiones están regidas por una normatividad propia que impone marcos de referencia extraordinarios, con respecto al exterior, que afectan a la observación y a la interacción entre investigador e investigado. La rutina, la espera, la desaceleración del tiempo ${ }^{4}$ de vida y la vigilancia permanente conforman un espacio de control directo y manifiesto y al que se subordinan los mínimos comportamientos, que hay que comprender cuando se investiga en su interior. Si la observación empírica sociológica puede considerarse en sí misma un dispositivo de control (Ibáñez 1979) en el contexto del control más o menos difuso y complejo que se desarrolla en las sociedades desarrolladas ${ }^{5}$, la observación en la cárcel entra a formar parte de un sistema de control manifiesto.

Ahora bien, a la hora de diseñar una investigación en la cárcel, como en cualquier estudio científico, la justificación que debemos encontrar es la vinculación entre nuestros presupuestos de investigación (preguntas, hipótesis y objetivos) y el contexto de privación de libertad. Es decir, por qué puede llegar a resultar interesante, adecuado e incluso imprescindible realizar la investigación en un espacio tan condicionado. Busquemos la respuesta.

Desde una perspectiva criminológica, cuando los objetivos de la investigación buscan la identificación y explicación de los procesos criminógenos mediante el trazado de trayectorias delictivas, la indagación de contextos sociales y culturas criminógenas o la aproximación a profesionales del delito, la cárcel

${ }^{4}$ Concepto tomado de Rosa y Scheuerman (2009). Sintomáticamente extendido en otras formas de vida: desempleados, sin vivienda, jubilados.

5 Para las sociedades capitalistas avanzadas, véase desde Marcuse (2010) a Basaglia y Ongano (2010), pasando, claro está, por el propio Foucault (2000). 
es uno de los espacios más adecuados. Las ventajas son las siguientes: los informantes se encuentran en una situación controlada, se dispone de los registros de su actividad delictiva y del tiempo necesario para planificar la observación. En estas circunstancias, la utilización de prácticas de observación cualitativa como las historias de vida, entrevistas, autoinformes o grupos focalizados es viable. Entre los inconvenientes, hay que considerar que el hecho de centrarse en los que están en la cárcel supone hacerlo en una parte de los actores, los que han sido puestos bajo la tutela del sistema penitenciario, los que -si se nos permite la expresión- han terminado su ciclo: tendencia a la comisión de delitos, comisión de los mismos, identificación y detención, juicio y prisión. Quedan fuera los que, por distintos motivos, dignos en cada caso de estudio, no cometieron delitos acumulando diversos factores y condiciones para tender a ellos, o, si lo cometieron, no fueron identificados y detenidos para cumplir con medidas sancionadoras de privación de libertad.

Desde una perspectiva sociológica, la justificación viene dada cuando el objeto de la investigación es la propia cárcel; y el objetivo, la evaluación del grado de cumplimiento de las funciones (manifiestas / latentes) de la institución. Así, cuando nos proponemos observar la vida en la prisión y sus efectos sociales, los procesos de agrupación y resocialización en la misma, la evaluación de los programas de rehabilitación o del servicio público que presta el sistema, la relación entre lo que sucede dentro de la cárcel y lo que sucede fuera en el entorno social del interno o los dispositivos de resistencia que generan los sujetos frente a la acción del sistema de castigo, la utilización del espacio penitenciario está justificada.

El uso de esta perspectiva requiere de una ampliación de las formas de obtener y producir información. La evaluación de programas puede ser realizada mediante diseños experimentales o cuasi experimentales; la vida en prisión puede ser observada mediante prácticas cualitativas; la evaluación del servicio público de prisiones mediante encuestas, grupos focalizados y análisis de documentos; $\mathrm{y}$, las relaciones interior / exterior mediante entrevistas.

A diferencia de la perspectiva criminológica, la variedad de categorías de informantes se amplía. Además de los reclusos, los funcionarios, gestores, profesionales de la intervención y la gestión, o los amigos y familiares, pueden verse solicitados como informantes.

Las ventajas metodológicas de la utilización de la cárcel para esta perspectiva son similares a la perspectiva criminológica: el acceso a unos sujetos que están en un espacio vigilado (dominado por la lógica del control y la seguridad), así como acceso a registros y disposición de tiempo para el diseño de investigaciones longitudinales.

Sin embargo, hay que contemplar también los problemas y limitaciones metodológicas que trae consigo este espacio. Además del problema ya mencionado de representatividad y sesgo en la selección de los informantes para los estudios criminógenos, hay problemas comunes a la mayor parte de estudios en este espacio: a) la elevada reactividad, que puede llegar al rechazo a la participación en la observación; b) la presión para la producción de indicadores generados 
dentro del espacio de control de la cárcel; c) los problemas que derivados de los bajos niveles educativos y de formación de los informantes, condicionan especialmente cuando se trata de instrumentos de recogida de información auto administrados; d) hasta los propios espacios facilitan poco la interacción entre investigadores y participantes en la observación, debiéndose tener en cuenta se trata de una práctica de control -la observación- en un espacio diseñado para el control.

Aunque neutralizar todas estas circunstancias, limitaciones y efectos es tarea práctica imposible, hay que seguir empeñados en encontrar una buena guía de actuación para reducir los posibles sesgos y errores. De momento, consideremos dos principios. El primero, orientar nuestro trabajo según el mantenimiento de un estricto respeto por los procedimientos de producción de evidencia empírica admitidos por las ciencias sociales. El segundo, mantener en todo momento un comportamiento ético y socialmente responsable.

\section{REQUERIMIENTO ÉTICO Y SOCIALMENTE RESPONSABLE}

Las exigencias éticas publicadas en los códigos deontológicos de las organizaciones profesionales más representativas de la sociología y la criminología ${ }^{6}$ en España necesitan una actualización. En la publicación actual, ninguna de ellas entra a valorar ni da recomendaciones específicas para las situaciones de especial vulnerabilidad, como la que se da en la cárcel u otras instituciones totales. Sus indicaciones son generales y se limitan, en el caso de la sociología, a la regulación de la integridad profesional (apartado a) y la integridad de los grupos o sujetos de estudio (apartado b). Aunque en estos apartados se habla del mantenimiento del secreto profesional, de las declaraciones que el sujeto de estudio realiza durante la investigación, la garantía del anonimato en las publicaciones, el consentimiento informado, la forma de aplicarlo y la idoneidad entre las técnicas utilizadas y las características de las personas que son investigadas. En conjunto, lo publicado es claramente insuficiente para responder con garantías a la complejidad que plantea un contexto que provoca una situación de especial vulnerabilidad. En el caso de la criminología, el compromiso es similar o más escaso aún. Llama la atención que enuncie la exigencia de trabajar dentro de los límites jurídicos, una cuestión que no es un compromiso ético sino una exigencia legal. Algo más concreto y actual es un buen apunte relacionado con la protección de los sujetos en el que se menciona la salvaguarda integral del investigado ${ }^{7}$. Ese es un buen camino.

${ }^{6}$ Código deontológico del Colegio Oficial de Politólogos y Sociólogos publicado en http:// colpolsoc.org/wp-content/uploads/2013/11/CodigoDeontologico3.pdf Revisado el 03 de diciembre de 2018. El Código deontológico de la Sociedad Española de Investigaciones Criminológicas está publicado en: https://seicdifusion.files.wordpress.com/2016/08/codigo deontologico.pdf Revisado el 03 de diciembre de 2018

7 Código Deontológico de la SEIC: "Durante el desarrollo de la actividad empírica, los investigadores deben asegurarse de que los sujetos no correrán riesgos que perjudiquen su bienestar 
Si observamos otros códigos éticos de organizaciones similares en otros países, la opción más actualizada y desarrollada es la que ha elaborado la British Sociological Association ${ }^{8}$ en 2017. En su código ético entra a orientar en profundidad el comportamiento y la toma decisiones de los investigadores en estas situaciones concretas. La responsabilidad de asegurarse que la investigación no afecte negativamente al bienestar físico, social y psicológico o el apartado sobre la investigación con grupos en situación de vulnerabilidad sería imprescindible trasladarla a nuestros códigos disciplinares.

También es interesante hacer un apunte sobre el desarrollo de códigos éticos transnacionales dadas las condiciones que produce la globalización, tanto en lo que se refiere al trabajo de equipos transnacionales como las cuestiones de publicación digital global. En este campo, The World Conferences on Research Integrity ${ }^{9}$ es la organización internacional que está intentando armonizar todas estas cuestiones. Los principios que proponen en sus trabajos son una buena guía de actuación general para equipos y editores de revistas transnacionales. ¿Y, qué debemos tener en cuenta mientras tanto?

Nuestra experiencia práctica nos permite realizar algunas recomendaciones básicas. La primera cuestión que debemos considerar antes de poner en situación de observación a cualquier persona privada de libertad es si el resultado de nuestro trabajo va a mejorar realmente sus condiciones de vida, la ejecución penal o va a facilitar los procesos de inserción o abandono del delito. Es decir, si nuestra trabajo busca mejorar su situación. Si no es así, lo mejor es parar en ese momento y buscar una estrategia para responder a nuestros interrogantes que al menos no le implique. En este sentido, debe haber una reflexión profunda y constante sobre el interés de la demanda explícita e implícita de investigación por parte de la institución penitenciaria u otras instituciones u organizaciones involucradas en este espacio.

Si la respuesta a la pregunta anterior es positiva, la segunda cuestión que debemos considerar es que nuestro trabajo tenga el menor efecto posible en la vida de los observados. En el espacio penitenciario, no se debe perder nunca el punto de vista reflexivo sobre la metodología y el trabajo empírico que estamos realizando. Además de ser especialmente respetuosos con las personas privadas de libertad, hay que tener en cuenta las posibles consecuencias negativas que nuestras prácticas de investigación introducen en el medio, incluso cuando no participan en la investigación. En ocasiones, el rechazo legítimo de los privados de libertad a participar en una investigación puede ser observado por algunos responsables de la institución penitenciaria como un comportamiento negativo, un reflejo de comportamiento resistente. Para evitar esa etiqueta institucional puede ocurrir que algunos acepten la participación como una obligación, sin

\footnotetext{
físico, social o psicológico. Especial atención y cuidado habrá que observar ante sujetos especialmente vulnerables por su edad o por cualquier otra circunstancia o condición.” Pág. 2.

${ }^{8}$ El código ético de la British Sociological Association es accesible en:

https://www.britsoc.co.uk/media/24310/bsa statement of ethical practice.pdf

Revisado el 03/12/2018.

${ }^{9}$ Página web: https://wcrif.org Revisada el día 3/12/2008
} 
estar de acuerdo. Por ese motivo, hay que ser especialmente cuidadoso en lo que a los procesos de selección se refiere. En nuestra experiencia profesional hemos visto rechazadas muchas peticiones de colaboración y eso es una situación que se debe evitar comunicar a la institución o si no hay más remedio hacerlo como algo normal, habitual, dentro de lo esperado y como un derecho. Hay que evitar que parezca un desafío a nuestra autoridad o un problema para nuestro trabajo. Hay que intentar por todos los medios que el proceso de selección de los participantes lo realicemos nosotros y que la institución participe lo mínimo posible. El consentimiento informado lo debemos gestionar directamente, sin intermediarios. Hay que evitar que la institución transmita los objetivos ni el objeto de la investigación. La solicitud de colaboración comunicada por la institución para la primera selección -es casi imposible que ese primer paso nos dejen darlo a nosotros- debe plantearse a partir de características generales, hay que evitar la petición de personas concretas. En la medida de lo posible, hay que asumir el trabajo de campo personalmente, entrar en la cárcel y controlar este y otros procesos. Si se envía un cuestionario (práctica habitual) para que los rellenen sin nuestra presencia ni nuestro control del proceso de selección directo, es posible que se pueda perjudicar a las personas que nos responden. En síntesis, el proceso de participación y el consentimiento informado es una tarea que no se puede poner en manos de la institución penitenciaria ni de sus técnicos, por muy buena voluntad y ganas de ayudar que pongan. No sólo hay que garantizar que la participación es libre, hay que garantizar que el rechazo a la misma no tiene consecuencias negativas.

La tercera cuestión a considerar es entender que nuestra posición respecto a los participantes es siempre de poder. Todo el que llega de fuera a observar es alguien que ofrece información explícita o implícita a la institución. Por eso, hay que ser extremadamente precavidos con nuestro comportamiento, con la forma en que se establecen nuestras relaciones, con quién hablamos y de qué, con no desvelar nuestras fuentes y nuestros resultados, tanto durante el trabajo de campo como en la publicación posterior. En este sentido, hay que tener en cuenta que nuestras publicaciones siempre pueden tener un efecto negativo sobre nuestros informantes.

Aprender a vigilar los posibles efectos indeseados de nuestras prácticas es tan importante como el respeto a los principios éticos o a los códigos deontológicos y es mucho más complejo de asegurar. Hay que mantener una mirada crítica a todas nuestras acciones. Hay que saber parar cuando se vislumbre el más mínimo riesgo para quienes participan o para los que nos apoyan. El espacio nos compromete a todos.

La institución penitenciaria solo nos exige el permiso de investigación y el consentimiento informado para realizar nuestro trabajo. De nuestra parte queda actuar con rectitud e integridad moral y respetar a todas las personas que de un modo u otro confían en nuestro trabajo porque creen legítimamente que su ayuda va a servir para mejorar su vida o la de otros que se encuentran o encontrarán en la misma situación. 


\section{EL SISTEMA PENITENCIARIO EN ESPAÑA}

Para investigar en la cárcel hay que conocer en profundidad sus características fundamentales. El primer paso para avanzar en ese conocimiento lo proporciona la estructura organizativa del sistema penitenciario, las condiciones de vida que rigen los distintos contextos de encierro, los roles que ejercen los distintos tipos de técnicos y funcionarios, las formas de ejecución de la pena de privación de libertad, las claves principales de la subcultura carcelaria o las características básicas de la población encarcelada. Una parte de esta información está disponible en las Guías que el sistema penitenciario publica en su web, otra se encuentra en el material empírico publicado y las obras de referencia que citamos en este documento u otras que pueden encontrar si siguen el hilo de la bibliografía; el resto lo acumularán con la práctica y experiencia de investigación.

Las competencias de la gestión penitenciaria en España las tiene el Ministerio del Interior. La ejecución de sus políticas depende de la Secretaría General de Instituciones Penitenciarias ${ }^{10}$ y la Direcció General de Serveis Penitenciaris ${ }^{11}$, responsable del sistema en Cataluña. Esta comunidad tiene transferidas las competencias del sistema penitenciario desde 1982. La Secretaría General se ocupa del resto del Estado. Aproximadamente entre un 14\% y un 16\% de los presos están encarcelados en Cataluña. Esta primera división institucional es muy importante a la hora de planificar cualquier investigación ya que la petición de permisos para investigar se deben dirigir a una u otra administración en función del ámbito territorial de su competencia. Esta división provoca que la gran mayoría de los trabajos de investigación que se realizan en España se refieran exclusivamente a uno u otro espacio territorial. La solución a esta limitación es conformar equipos con experiencia en una y otra administración y trabajar conjuntamente.

También hay que considerar esta diferencia a la hora de manejar los datos sobre población penitenciaria que publican organismos públicos como el Instituto Nacional de Estadística y comprobar la base territorial a la que se refiere. Esta es una de las fuentes de errores más comunes cuando se habla de la "población penitenciaria en España".

La normativa legal que regula la actuación para ambas administraciones es la misma ${ }^{12}$. La administración catalana ha desarrollado una normativa específica para dotar de marco legal al Centre d'Iniciatives per la Reinserció (CIRE) y otras actuaciones ${ }^{13}$ menores de carácter organizativo. En Cataluña, la administración penitenciaria está adscrita al Departament de Justícia.

${ }^{10}$ Web de la Secretaría General de Instituciones Penitenciarias. Revisado enero 2108. http://www.institucionpenitenciaria.es

${ }^{11}$ Web de la Direcció General de Serveis Penitenciaris. Revisado enero 2108. http://justicia.gencat.cat/ca/ambits/reinsercio i serveis penitenciaris/

${ }^{12}$ Régimen jurídico. Revisado enero 2108 http://www.institucionpenitenciaria.es/web/portal/ administracionPenitenciaria/normativa.html

${ }^{13}$ Régimen jurídico específico de Cataluña. Revisado enero 2108. http://justicia.gencat.cat/ca/departament/Normativa/normativasectorial/penitenciaria/ 
La estructura de los Servicios Centrales de la Secretaría General recae sobre dos instituciones que actúan con bastante independencia: la Entidad Estatal de Derecho Público, Trabajo Penitenciario y Formación para el Empleo (antiguo Organismo Autónomo de Trabajo y Formación para el Empleo) y la propia Secretaría General, que alberga 7 Subdirecciones Generales que abarcan todas las áreas de actuación del sistema penitenciario. Aunque pueden variar algo en función de la organización, los campos de trabajo son los mismos en ambas administraciones.

\section{Cuadro 1: Estructura Servicios Centrales de la SGIIP.}

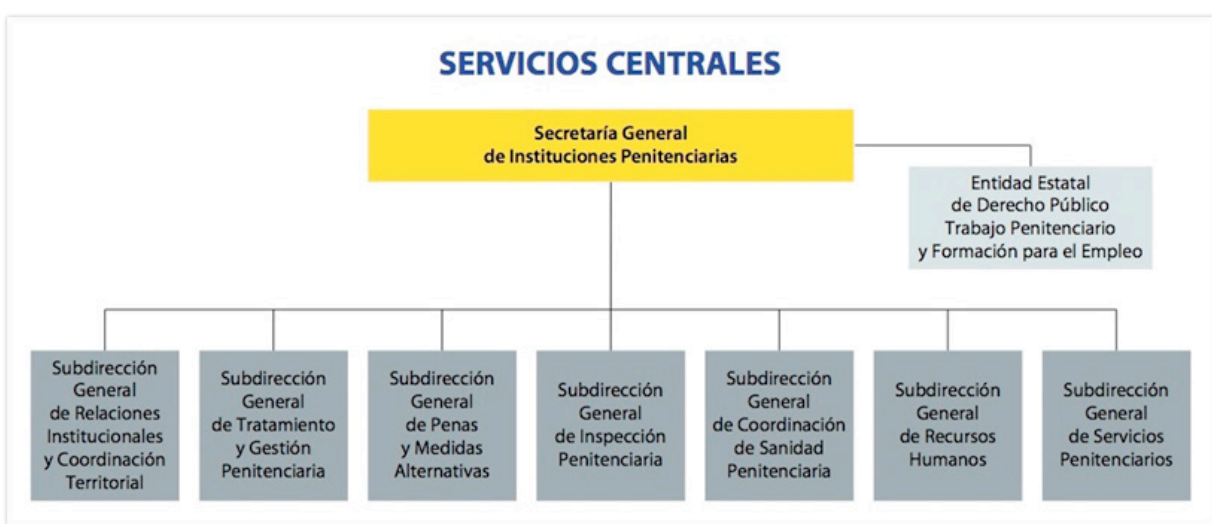

Tomado de la Guía del Sistema Penitenciario Español publicada por la Secretaría General de IIPP en su página web.

La Subdirección General de Relaciones Institucionales y Coordinación es la puerta de acceso a la comunicación para la formalización de petición de proyectos de investigación. También, esta es la Subdirección con la que nos comunicamos para gestionar los permisos de acceso y trabajo en los distintos tipos de centro. No obstante, aunque se obtengan los permisos. Contactar con el centro penitenciario o el CIS antes de hacer la visita es una buena forma de evitar determinantes problemas de acceso.

A efectos de investigación, todos los estudios que se refieran al trabajo y a la formación para el empleo deberían considerar a la Entidad Estatal como referencia de información, aunque los permisos de investigación dependan en cualquier caso de la Dirección de la Secretaría General. En Cataluña, el organismo que realiza esta función es el CIRE. Aunque es complejo conseguir la información, las fuentes de datos de esta entidad son muy buenas. Aunque es difícil, debemos intentarlo. La información sobre empleo, estructura laboral, el reparto del trabajo, contrataciones etc.. están digitalizados y actualizados continuamente.

La Subdirección General de Tratamiento y Gestión Penitenciaria es la responsable de la organización y gestión de dos ámbitos clave para la investigación: la educación para adultos y la formación profesional, y los programas de inter- 
vención externos e internos. Todas las organizaciones del tercer sector e instituciones que trabajan dentro del sistema se coordinan desde esta subdirección. La educación básica depende de las consejería de las CCAA y la superior, de la UNED. Todos los programas específicos (violencia de género, agresores sexuales, prevención del suicidio, etc...) son coordinados desde esta subdirección. En consecuencia, cualquier trabajo de investigación que tenga relación con la intervención social y los procesos de inserción debe considerar esta subdirección como una buena fuente de información.

La Subdirección de Penas y Medidas Alternativas es la que gestiona las penas que no tienen como consecuencia el ingreso en prisión. En el argot, son los que gestionan la ejecución de las penas de los TBC (Trabajos en Beneficio de la Comunidad) y los programas de reeducación que están sustituyendo a los TBC. La gestión de estas sentencias se llevan a cabo en una sección específica de los CIS (Centros de Inserción Social), en "Medio abierto". Este es una campo de la investigación apenas explorado en España.

La Subdirección General de Inspección Penitenciaria tiene una misión de control y evaluación interna, pero también realiza estudios sobre el delito muy interesantes. El problema es que los resultados de sus trabajos tienen una difusión interna. A veces, hay alguna publicación suya en la web de la Secretaría General. Se puede explorar en la zona de publicaciones. En la actualidad, hay algún trabajo sobre reincidencia.

La Subdirección General de Coordinación de Sanidad Penitenciaria es la responsable de todos los trabajos y servicios relacionados con la salud en el ámbito penitenciario. Publican datos sobre mortalidad, enfermedades y salud en los informes anuales. Los trabajos de los programas sobre salud mental PAIEM (Programa de Actuación de Internos con Enfermedad Mental), una cuestión social clave, se gestionan desde esta Subdirección. Hay varios informes publicados en la web de la Secretaría General sobre el asunto de las enfermedades mentales de enorme interés. En este campo, también la Revista Española de Sanidad Penitenciaria publica artículos específicos.

La Subdirección General de Recursos Humanos es la que se encarga de la gestión del personal de la institución. Hay poca información disponible y poca investigación realizada en España sobre estos trabajadores. Los datos básicos sobre personal se publican en los informes generales anuales disponibles en la zona de publicaciones de la web de la Secretaría.

En Cataluña, la organización no es muy diferente, pero sí hay que reseñar, por su especial interés para la investigación criminológica, la actividad que realiza el CIRE. Esta institución está desarrollando una serie de valiosos trabajos sobre campos pocos explorados en España. El desistimiento, los procesos de reingreso y la integración de ciudadanos en el ámbito de la intervención son los que tienen más interés en la actualidad (los estudiantes pueden buscar los trabajos del profesor Josep Cid Moliné y las publicaciones del CIRE en las publicaciones del Departament de Justícia y en la web de la Secretaría General de Instituciones Penitenciarias) 
Descendamos un escalón en la estructura para explicar los tipos de establecimientos que constituyen el sistema ${ }^{14}$. La condena de privación de libertad se ejecuta en varios tipos de establecimientos: los Centros Penitenciarios Ordinarios (CPO), los Centros de Inserción Social (CIS), las Unidades de Madres, las Unidades Dependientes y los Hospitales Psiquiátricos Penitenciarios. Las diferencias entre unos y otros son muy grandes. A la hora de decidir el diseño de una investigación, la elección del tipo de establecimiento es una cuestión clave. Veamos las características y las posibilidades de investigación.

En los Centros Penitenciarios Ordinarios (CPO) se encuentra la mayor parte de la población penitenciaria. En estos centros, los presos cumplen casi toda su condena, incluso pueden llegar a cumplirla íntegramente. Las instalaciones de los CPO son las que tienen un régimen de contención y seguridad mayor.

En los CPO conviven preventivos (en espera de juicio o condena) y penados (cumpliendo sentencia). Los primeros no son clasificados hasta que la sentencia no se produce. Este tiempo de espera -a veces años- es un período de incertidumbre y tensión que puede afectar al comportamiento de los sujetos. Hay que tener en cuenta también que la dependencia económica de la ayuda externa de los preventivos es mayor porque no pueden conseguir empleo dentro del sistema penitenciario en esa etapa. Además, es la primera fase de aislamiento social y en el caso de los extranjeros la situación, si cabe, es mucho más complicada. Hay que considerar también que son periodos de adaptación a un régimen de vida muy estricto. La situación de los primarios siempre es más delicada. Cuando se seleccionan los sujetos que van a participar en la investigación, es importante hacer una reflexión sobre los posibles efectos de estas cuestiones.

Una vez condenados, los presos son clasificados según criterios penales (condena, actividad delictiva, tipo de delito..), criterios penitenciarios (conducta penitenciaria y conducta global), criterios científicos (psicológicos, sociológicos y criminológicos) y otros (integración) Fernández y Nistal (2012:458). Las tres categorías resultantes son: primer grado (mayor contención y mayor seguridad) (Ríos Martin, J. C y Cabrera, P.J. 2002), segundo grado (régimen ordinario) y tercer grado (menor contención, en ocasiones semilibertad). En los CPO, la mayor parte de los presos están en segundo grado. Los que están en primer grado suelen estar en régimen cerrado, aislados y es casi imposible acceder ${ }^{15}$ a ellos. Pero no hay que olvidar que esta situación es transitoria y que hay presos clasificados en segundo o tercer grado que han podido estar durante algún tiempo en primer grado. Es decir, esos períodos y sus efectos también se pueden investigar cuando mejore su situación. En los CPO, hay pocos presos que estén en tercer

${ }^{14}$ Comprender con mayor precisión la terminología y el anclaje jurídico de muchos conceptos y tecnicismos habitualmente utilizados, al investigador le convendría utilizar cualquier manual comentado de Derecho penitenciario. En estos manuales podrá encontrar muchas claves para comprender el campo. Uno de los mejores es el de Fernández y Nistal (2012). Así mismo, también pueden resultar adecuados los de Cervelló (2016) o Ríos Martín y otros (2018).

${ }^{15}$ Los trabajos de Eugenio Arribas López sobre el régimen cerrado y los primeros grados, publicados en la Revista General de Derecho Penal pueden ser un punto de partida para iniciar estudios en este campo. 
grado, tan sólo los que no tienen red de apoyo externo (extranjeros y, sobre todo, mujeres extranjeras desarraigadas). Lo habitual es que los que están en tercer grado pasen a las Secciones Abiertas o a los Centros de Inserción Social en un régimen de semilibertad.

En la mayoría de los CPO, hay uno o dos módulos de mujeres. El resto son módulos de varones. Lo habitual es que las mujeres sean entre el 8-10\% de la población total del centro. El mayor problema del encarcelamiento de las mujeres que cumplen condena en los CPO es que esos centros están pensados para varones, en todos los sentidos. La seguridad y el aislamiento son excesivos para su nivel de peligrosidad. El acceso a las actividades del centro es más difícil para ellas. Las desigualdades con los varones son importantes. En los módulos de mujeres, no hay posibilidad de separación entre las "primarias", reincidentes, preventivas y condenadas, jóvenes y mayores o las de mayor y menor peligrosidad. Esta diversidad afecta a la intervención y a la convivencia cotidiana. El campo de los estudios de género en prisión se ha ido desarrollando lentamente; pero con buenos resultados. En España, los trabajos sobre mujeres presas de la profesora Elisabeth Almeda $(2002,2017)$ y los realizados por los autores de este artículo (Del Val y Viedma 2012) ${ }^{16}$ pueden ser interesantes referencias para iniciarse en su estudio. El trabajo de Pat Carlen ${ }^{17}$ y la criminología crítica feminista es un camino obligatorio para recorrer.

\section{Cuadro 2: Estructura organizativa de los Centros Penitenciarios Ordinarios.}

\section{CENTRO PENITENCIARIO}

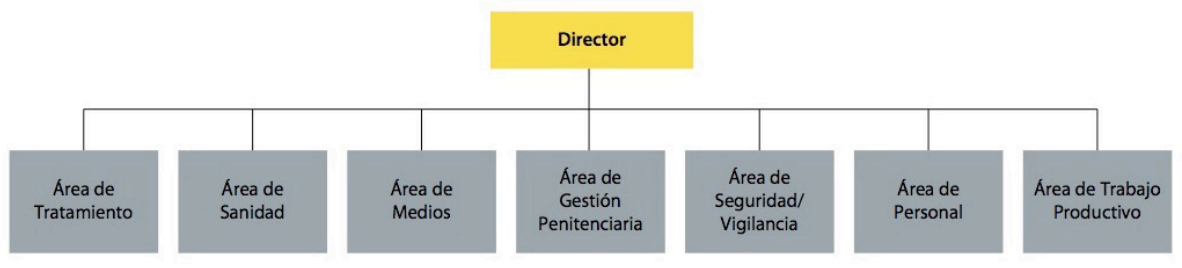

Tomado de la Guía del Sistema Penitenciario Español publicada por la Secretaría General de IIPP en su página web.

En la estructura organizativa de los CPO, la dirección cumple un papel clave. De ella dependen jerárquicamente todas las áreas del CPO y la jerarquía es

${ }^{16}$ El libro Condenadas a la desigualdad es un estudio sobre las situaciones de desigualdad que se producen con las mujeres encarceladas en España que aborda la creación de un sistema de indicadores de desigualdad. (Del Val y Viedma 2012)

${ }^{17}$ El trabajo de Pat Carlen es una referencia imprescindible para cualquier investigación que se aproxime a la situación de las mujeres en prisión. Pagina we de su obra: https://www2.le.ac.uk/ departments/criminology/people/professor-pat-carlen/professor-pat-carlen-publications 
el modo en que se construye la organización. El Director o Directora asume el poder y el liderazgo del CPO y su modo de comprender la acción penitenciaria se transmite a todas la áreas. Es lógico que todos ellos tengan una preocupación mayor por la seguridad, pero la clave de la cultura que imprimen en el centro está en su concepción del castigo y en los fines de la cárcel. El equilibrio entre seguridad y reinserción es la medida de la convivencia que generan y este es un indicador para saber cuál es la cultura del centro. Para nosotros una fuente para evaluar la situación.

Este puesto de dirección en el CPO es la única figura de libre designación, es decir, su nombramiento se produce en la Secretaría General. Es un cargo con un marcado cariz político. Los otros miembros del equipo directivo son nombrados a propuesta del director. Para muchos, porque apenas hay mujeres, la dirección es la culminación de su carrera profesional en los CPO. Cuando acaban pueden pasar a ocupar puestos en la Sede Central, volver a sus antiguos puestos técnicos o quedar como "Directores de Programas", un cargo casi simbólico en los CPO. De cualquier manera, ellos tienen la llave de acceso. Ellos son nuestros "porteros del campo". Sin su apoyo es imposible trabajar. Lo habitual es que cuando se plantea una investigación en un CPO la dirección haya mostrado su consentimiento por participar. De cualquier modo, siempre hay que considerar la cultura de centro que promociona la dirección.

Los responsables de las áreas más relacionadas con la investigación social (tratamiento y trabajo) son técnicos pero con diferente nivel y mando. La Subdirección de Tratamiento suele estar a cargo de un psicólogo/a (aquí hay más mujeres), mientras que el Área de Trabajo Productivo está a cargo de un funcionario de menor nivel profesional (Coordinador laboral, no olvidar que el trabajo productivo depende de la Entidad Estatal). Si la dirección nos abre la puerta del exterior, la subdirección de tratamiento nos la abre del interior. Todas las actividades, programas e intervenciones dependen de este área. La educación, el deporte, el entretenimiento, la intervención psicológica y la asignación y clasificación de los presos para el trabajo está bajo su mando. Podríamos decir que gestionan la convivencia y éste es un factor clave. Son los más receptivos a la investigación social externa y los que nos pueden ayudar en la gestión del trabajo de campo en el interior. De hecho, suelen ser en los que delega la dirección para ayudarnos.

El área de sanidad está dirigida por médicos que son funcionarios de la administración penitenciaria. Esta dirección es clave no sólo en la salud, sino en la salvaguarda de los derechos de los reclusos. En sus manos, está la elaboración de informes de lesiones, suicidios y muertes. El control médico de los presos y, especialmente, de los de régimen cerrado, la intervención en salud mental y su relación con los Jueces de Vigilancia, son factores clave para la organización y la relación entre funcionarios y presos. El área sanitaria es un campo de especial relevancia que no se investiga suficiente (véase la explicación publicaciones de la subdirección que es responsable de la salud penitenciaria)

Cuando se realiza trabajo de campo en el interior de los CPO, los espacios que habitualmente se utilizan son los de la zona cultural. Es el lugar más accesi- 
ble y el único donde se garantiza la intimidad suficiente como para poder grabar una entrevista o rellenar un cuestionario. Es decir, el lugar donde poder crear esa particular característica del espacio de observación, entre una especie de espacio privado dentro del espacio público y una especie de espacio público -ya que la observación genera publicidad, espacio público- dentro del espacio que el informante considera privado. Las aulas del "socio" son las más adecuadas porque son las que están menos cargadas simbólicamente. Si nos fijamos en el cuadro 3 , "el socio" es el edificio que alberga la escuela y el salón de actos. Está en el interior del tercer anillo de seguridad.

Hay que tener en cuenta que los presos que vayan a participar tienen que salir de los módulos y ser conducidos hasta este edificio. Es decir, tendrán que tener una orden firmada por la dirección o la jefatura de seguridad para ser trasladados del módulo al socio. Estos traslados hay que solicitarlos y justificarlos con tiempo. La mayor complicación que tiene utilizar este espacio es la temporal. En la práctica, este espacio sólo se puede usar por las mañana y ese es el momento en que más actividades se programan. Por este motivo hay que planificar el uso de espacios y eso también hay que hacerlo con antelación. El coordinador de formación y la subdirección de tratamiento suelen ser las figuras que pueden conseguir esos espacios.

Antes de acabar con este espacio recordaremos algunas cuestiones clave: para entrar en un CPO, hay que ir siempre identificado con el DNI. El acceso se debe haber gestionado con antelación en la Secretaría General. Cuando lleguemos al CPO, tiene que haber un permiso en el control de accesos para poder entrar, especificando la zona que se va a visitar y la actividad que se va a realizar. Es evidente que para poder realizar cualquier trabajo de investigación en el interior hay que tener una persona que nos acompañe. ¿Cuál es el grado de mediación de este tercer actor en el proceso de interacción con los informantes? Difícil calibrarlo; pero existe.

Siempre que se va a un centro por primera vez, el lugar en el que debemos presentarnos es en la dirección. De hecho, esa primera reunión será crucial para el desarrollo del trabajo. La dirección es nuestro portero del centro. Hay que preparar un guión lo más accesible posible para explicar el trabajo que vamos a realizar, sus objetivos y, sobre todo, lo que vamos a necesitar (espacio, tiempo, instrumentos de registro, etc.). Si se quieren introducir grabadoras para la entrevista hay que tener en cuenta que sólo pueden grabar sonido y que hay que pedir permiso en la Secretaría General. Los móviles están prohibidos en el interior. También hay que tener en cuenta el estricto horario que existe. Hasta las 10 no hay casi posibilidad de hacer nada porque los presos están en los módulos. A las 13:00 van a comer. Y por la tarde, es prácticamente imposible.

Pasemos al segundo tipo de establecimiento, los Centros de Inserción Social. El contexto de cumplimiento en régimen de semi libertad se produce en los CIS. Estos establecimientos penitenciarios están destinados al cumplimiento de las penas privativas de libertad en régimen abierto (la legislación de este régimen se desarrolla en el capítulo 3 del Reglamento Penitenciario). De forma mayoritaria, en los CIS cumplen condena las personas que están clasificadas en tercer grado. 
Esta clasificación permite salir del CIS diariamente para búsqueda de empleo, realización de cursos u otras actividades que faciliten la inserción. En la práctica, estos sujetos son aquellos que la Institución Penitenciaria considera que se encuentran en un proceso avanzado de reinserción. También hay un grupo de clasificados en el artículo $83^{18}$ que son los que están en el CIS porque tienen un empleo durante el día y regresan a dormir por la noche. Con una presencia similar, los clasificados en el art. 86, llamados cotidianamente "telemáticos", cumplen condena siendo vigilados por una pulsera electrónica conectada al CIS que da cuenta de sus movimientos. Este grupo puede ir a dormir al Centro o hacerlo en su domicilio. En el CIS, también se realiza el seguimiento de los liberados condicionales, pero su presencia se limita al control mensual de su situación.

En definitiva, los sujetos que cumplen condena en los CIS reúnen una serie de condiciones que facilita su selección. La proximidad a la libertad y el hecho de hallarse en una situación cuasi experimental "dentro-fuera" convierte a estos establecimientos en espacios ideales para observar las estrategias configuradas por los reclusos en las fases inmediatamente previas a su excarcelación. En esta situación también se puede observar las potencialidades y limitaciones reales de los programas de tratamiento e inserción social, pudiendo contar con la participación de agentes externos a la prisión que trabajan para la inserción.

Hay una división significativa en estos establecimientos, hay CIS dependientes y CIS independientes. Los primeros son establecimientos que dependen funcionalmente de la dirección de un CPO. Es decir, la dirección formal del CIS es la del CPO. Los CIS independientes tienen una organización autónoma y dependen del Área de Medio Abierto de la Secretaría General. También son diferentes las tres Unidades de Madres abiertas en la actualidad y convertidas en CIS. También, hay cuatro secciones abiertas que, aunque con limitaciones, gestionan el régimen abierto desde el interior de los CPO.

La estructura organizativa y el modo de actuación de los CIS es muy similar al de los CPO. La dirección tiene un papel igual de relevante que en los CPO. La única diferencia es que aparece un área nueva, que es la que gestiona las penas y medidas alternativas. Esta oficina funciona de modo casi autónomo al CIS.

${ }^{18}$ El artículo 83 del Reglamento de la Ley Penitencitaria es el que se encarga de desarrollar el régimen abierto. 


\section{Cuadro 3: Estructura organizativa de los Centros de Inserción Social.}

\section{CENTRO DE INSERCIÓN SOCIAL}

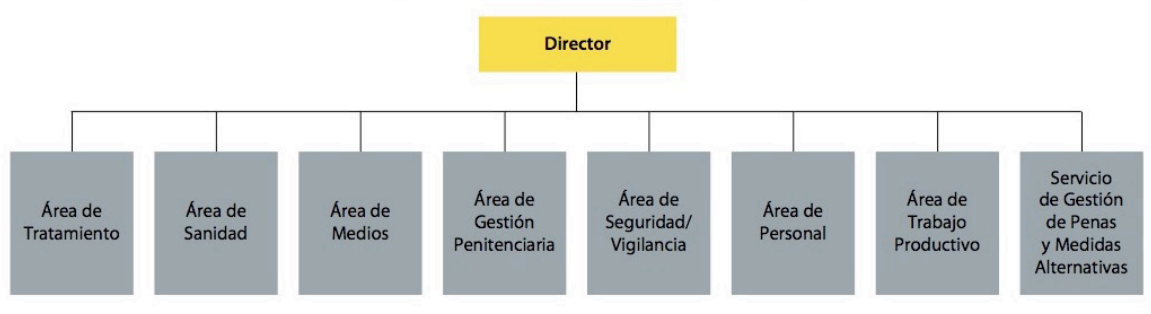

Tomado de la Guía del Sistema Penitenciario Español publicada por la Secretaría General de IIPP en su página web.

Las condiciones para el trabajo de campo en el CIS son distintas de las de los CPO. Aunque el acceso se consigue del mismo modo, al existir unos niveles de seguridad y control muy inferiores en el establecimiento, los movimientos en su interior son mucho más fáciles. La comunicación con los presos es más ágil y no hay tantas esperas. En el CIS todo el mundo tiene más libertad de movimiento. Ahora bien, la comunicación y el manejo de la interacción entre investigador e investigado es muy similar e igual de compleja.

El mayor problema del trabajo de campo en los CIS es la selección de los presos y encontrar el horario para poder contactar con ellos. Como la mayoría de los que están en los CIS salen a diario, encontrarlos dentro del establecimiento es complicado, sobre todo los liberados condicionales. Hay que buscar un horario para poder realizar el trabajo o diseñar una estrategia conjunta. De todos modos, la situación de control y seguridad en los CIS es mucho más grande que en los CPO. Sin duda, los filtros que va imponiendo la institución en la clasificación tienen sus efectos. Aunque no tienen que ser las mejores trayectorias delictivas, si son los que tienen un mejor comportamiento y actitud. Al menos, los que muestran un proceso de normalización mayor. Esta característica también es fundamental cuando se investiga en este espacio.

Esta zona intermedia no se ha estudiado apenas. Su relativo menor tiempo de implantación, ya que el $90 \%$ de los CIS no tienen apenas 10 años, no los ha dado a conocer. Sin embargo, por la manifiesta mayor accesibilidad como por su carácter trasnsicional, para los estudios de integración social es seguramente el mejor espacio.

Los otros tres tipos de establecimientos que existen tienen un peso muy reducido. Las Unidades de Madres, las Unidades Dependientes y los dos Hospitales Psiquiátricos Penitenciarios. Las Unidades de Madres son el último intento de sacar a los niños de los CPO. Hay tres establecimientos abiertos y dos pendientes de inauguración. Hay muy pocas mujeres viviendo en ellos. Las Unidades De- 
pendientes son pisos regidos por ONG en los que conviven presos que están en las últimas fases de su condena. Hombres solos sin arraigo o jóvenes con dificultades de integración. También están muy poco estudiados. Poco se conoce de los hospitales psiquiátricos y de lo que se hace en su interior. El estigma funciona.

\section{CARACTERÍSTICAS DE LOS ACTORES IMPLICADOS}

Uno de los primeros pasos que suele dar cualquier investigador social que pretenda conocer a una población es realizar un estudio inicial de sus características sociodemográficas. La forma en la que se distribuye una población según el sexo, la edad, la situación de convivencia, la clase social, su nivel de estudios, la nacionalidad, su descendencia, la situación de empleo, los ingresos etc.. puede ayudarnos a concretar una primera mirada exploratoria.

$\mathrm{Al}$ enfrentarnos a esta tarea, la primera cuestión a resolver es la disponibilidad de fuentes fiables de las que poder obtener la información que necesitamos o si, por el contrario, deberemos producir nuestros propios datos. Lógicamente, la cuestión sobre qué tipo de datos necesitamos, para qué y por qué son necesarios para conocer nuestro objeto de investigación son las cuestiones sobre las que se debe reflexionar a la hora de formular el proyecto de investigación.

Una vez revisadas las fuentes, casi siempre nos encontramos con que hay algunos datos disponibles y otros que deberíamos producir. La cuestión entonces es si, con los datos disponibles, podemos responder a nuestras preguntas de investigación. Si la respuesta es positiva, lo único que debemos hacer es recopilarlos, reelaborarlos según nuestro interés de investigación, analizarlos y publicarlos. Si los datos no son suficientes o inadecuados, se puede deber a varias cuestiones: o bien, no se han recopilado porque a la fuente que los produce no le interesan o no le resultan relevantes, en cuyo caso deberíamos producirlos; o bien, se han recopilado, pero se publican de forma agregada y no podemos indagar sobre cuestiones más concretas que resultan de nuestro interés. Por ejemplo, se pueden tener los datos de la distribución de la población penitenciaria por grupos de edad y de la misma población por nivel educativo; pero el cruce de las variables edad y nivel educativo no se publica. Si una de nuestras preguntas de investigación hubiera sido: ¿cuál es el nivel educativo de la población penitenciaria según grupos de edad? no podríamos responderla. Por no hablar de la imposibilidad de realizar análisis multivariantes. La solución, entonces, sería preguntar a la institución que publica la fuente si nos puede ofrecer los datos de los registros primarios de los individuos (microdatos), para poder hacer nosotros la explotación adecuada. Hay instituciones que, con las debidas precauciones -anonimizando los registros o exigiendo compromiso de confidencialidad al investigador- ofrecen estos datos, como hacen el Instituto Nacional de Estadística o el Centro de Investigaciones Sociológicas. Pero hay instituciones que no lo hacen (administraciones penitenciarias) y en este caso deberíamos producirlos nosotros. 
Con los datos que actualmente publica la Secretaría General se puede obtener un perfil básico de la población penitenciaria. Las variables que publica en su página web permiten conocer las siguientes características ${ }^{19}$ :

Según datos mensuales

- Población reclusa por sexo

- Situación procesal, personal, por sexo

- Grado de Tratamiento

- Distribución por Comunidades Autónomas

- Penados por grupos de edad y sexo

- Preventivos por grupos de edad y sexo

- Delitos Código penal derogado

- Delitos LO 10/1995 del CP

- Extranjeros por sexo.

- Penas y Medidas Alternativas

En los datos semanales se puede encontrar la evolución total de la población y la evolución por Comunidades Autónomas, el resto de las funciones no ofrecen datos.

Además de estas variables, en los informes anuales que publica en su fondo documental (accesible en su página web) se puede acceder a un gran número de datos referidos a todas la áreas de competencia de la institución: la situación penal, los tipos de delito, permisos, las actividades, la salud, el empleo, etc.

A grandes rasgos, la población penitenciaria es mayoritariamente masculina, solo alrededor de un $7 \%$ son mujeres, aunque la población de mujeres respecto a los países de nuestro entorno es del doble. Alrededor del 27\% son extranjeros, este dato ha bajado casi un 15\% desde el cambio del Código Penal de 2010. Entre las mujeres este porcentaje aumenta. Es una población relativamente joven, 2 de cada 3 son menores de 40 años. Con un nivel educativo muy bajo. Alrededor de un $7 \%$ se declara analfabeto al entrar, el $25 \%$ no acabó los estudios primarios, un $35 \%$ acabó la primaria, la primera etapa de secundaria, la que da acceso al graduado escolar lo consiguió el 20,7\% y la secundaria la concluyó el 11,2\%. El porcentaje de universitarios es de 3,2.

Además de los presos, hay que tener en cuenta que hay otros actores implicados en el campo que pueden ayudarnos en nuestra labor de investigación. Hoy día, alrededor de 1000 organizaciones trabajan en prisión en programas de intervención de todo tipo. Tienen contacto directo con la institución, con los presos y con los funcionarios. Con ellos se puede trabajar en el exterior. Sin duda, pueden ser una fuente de información muy valiosa. No hay apenas datos de quiénes son

19 Enlace a la página de estadísticas de la Secretaría General de Instituciones Penitenciarias. Revisado en enero de 2018.

http://www.institucionpenitenciaria.es/web/portal/documentos/estadisticas.html

Enlace a la página de estadísticas del Sevei penitenciari.

http://justicia.gencat.cat/ca/departament/Estadistiques/serveis penitenciaris 
estos voluntarios, ni sus características, podría ser un buen comienzo para abrir el campo de trabajo.

El tercer grupo de actores implicados en el campo son los funcionarios de prisión. Un cuerpo que supera los 25.000 trabajadores y que ha cambiado radicalmente en las últimas décadas. Si se hace una revisión de los informes generales y se observan los requisitos de ingreso se podrá inferir que su nivel técnico y de formación se ha elevado mucho respecto a aquellos funcionarios heredados de la transición que eran seleccionados por su adscripción política. A diferencia de los estudios que se realizan en el mundo anglosajón, la investigación sobre la situación de estos funcionarios es muy escasa. Otro campo de estudio pendiente.

\section{ESTRATEGIAS PARA INVESTIGAR EN LA CÁRCEL}

En la presentación del libro "Proyectos y Estrategias de investigación", hay unos párrafos en los que se ofrece una aproximación a los requerimientos para diseñar una buena estrategia de investigación. El párrafo que sintetiza la reflexión plantea lo siguiente:

Para las decisiones estratégicas, se requiere: a) un buen conocimiento del terreno... b) reconocimiento del estado de sus recursos; de las posibilidades para investigar en función del tiempo, dinero o habilidades disponibles, en este caso; y c) capacidad para leer la "situación de la batalla"... (Callejo y Viedma 2007: Prólogo IX)

Todas las cuestiones que se presentan son tareas necesarias y complejas que requieren de experiencia y conocimiento práctico. Ahora bien, si tuviéramos que valorar su efecto sobre la investigación, la capacidad para leer la "situación de la batalla" (proviene de la metáfora del estratega militar) es la más importante. Y el momento clave de esta lectura del campo se realiza cuando se diseña la investigación. En esa fase se decide la conformación del equipo de investigación, la definición de los papeles que van a cumplir las distintas instituciones implicadas, la elección de los espacios en los que se va a realizar en trabajo de campo, los sujetos a investigar y el diseño metodológico. Veamos cómo abordar estas decisiones estratégicas.

Sobre la conformación del equipo de investigación, todas las recomendaciones de los evaluadores de proyectos nos indican que los equipos multidisciplinares proporcionan una ventaja importante. En nuestro experiencia, la inclusión de sociólogos, juristas (penalistas), psicólogos y profesionales con experiencia laboral en cárceles produce resultados positivos. Las tres disciplinas y los profesionales tienen perspectivas teóricas y claves de interpretación que se complementan para cubrir aspectos que de otro modo no se abordarían.

Respecto al papel de las instituciones implicadas, en principio, lo habitual es que en una investigación en la cárcel participen una universidad u otra institución que provenga del mundo académico (fundaciones, institutos de investigación, observatorios etc.), profesionales de la intervención (ONG) o re- 
presentantes de grupos que ejercen algún control sobre estos espacios (Defensor del pueblo o similar, Instituciones de Derechos Humanos). En cualquier caso, el papel que asumen estas entidades es el de observadores independientes. La otra institución implicada es el propio sistema penitenciario. Aquí se hablará del primer caso, es decir, un grupo de investigación que proviene de una universidad y solicita la colaboración de la institución penitenciaria.

La pregunta a resolver es el papel que debe cumplir la Institución Penitenciaria (IP), su grado de participación y el nivel de implicación en el proceso de investigación. Tras varias investigaciones, las conclusiones a las que hemos llegado son las siguientes: en primer lugar, en la mayoría de los casos, aunque la academia y el sistema político son los destinatarios principales de nuestro trabajo, la IP es la más afectada por la investigación. Sea un documento crítico, amable o beligerante el interés de la IP es muy grande. Hay que eliminar los prejuicios y los estereotipos que nos impiden ver que dentro de la IP hay cientos de técnicos con un elevado grado de formación que quieren conocer los resultados de la investigación que realizamos. En segundo lugar, establecer un debate con la IP sobre el valor y la necesidad de nuestra investigación es necesario. Puede ser que nuestros planteamientos se alejen de sus posiciones, puede que abordemos temas teóricos que quizá no tengan una conexión directa con sus prácticas profesionales, pero lo que es indudable es que ese debate puede tener repercusiones positivas. En tercer lugar, establecer una colaboración pactada es el mejor modo de acceder al campo. Esa colaboración se puede limitar a tener un técnico apoyo de la institución que se encargue de establecer comunicaciones, facilitar el acceso y ofrecernos información relevante para la investigación. Es decir, un "portero institucional" de segundo nivel. También es posible aumentar el grado de participación e implicar al técnico en los procesos de investigación que no manejen información que pueda afectar a los informantes. En cuarto lugar, se puede realizar una investigación consiguiendo el permiso de acceso y entrando sin ninguna implicación de la IP. No conocemos estos casos. Ya sea con apoyos informales -algún técnico o funcionario que ayuda en la sombra- o dejándonos hacer sin rechazo, la IP siempre está condicionando el trabajo.

Sobre la elección de los espacios de observación, los sujetos y el diseño metodológico ya conocemos que estas tres cuestiones están íntimamente ligadas y condicionadas por las preguntas / hipótesis / objetivos que nos planteemos resolver con la investigación. En la cárcel es prioritario decidir sobre la necesidad de que el estudio se lleve a cabo en un centro o en varios, conocer el grado de accesibilidad a los sujetos que nos deben informar, las condiciones en las que se produce esa relación y sus características. La solución a estas cuestiones conforman un primer marco estratégico de decisión. Intentemos obtener claves para su solución.

Hay muchas investigaciones que se llevan a cabo en un solo centro penitenciario. Lo adecuado de este diseño no se puede justificar por la facilidad de acceso, el ahorro de esfuerzos o cualquier razón instrumental ni económica. La clave de la elección debe estar en la ejemplaridad motivada que se pueden alcanzar con los resultados de ese centro concreto respecto de nuestro objeto de estudio. 
Es decir, con un solo centro no se puede plantear representatividad estadística o sociológica alguna, a lo único que podemos aspirar es a encontrar un ejemplo de una situación, grupo, programa, intervención especial o cualquier otro acontecimiento que por algún motivo esté representado ejemplarmente en ese centro, esto es, a un estudio de caso (Yin 2009; Coller 2000). Y a través de ese estudio concreto, buscar la comprensión de lo que sucede. Por ejemplo, cuando se comenzaron a utilizar las primeras cárceles modulares, los estudios sobre los efectos de aislamiento en los presos se podían haber llevado a cabo en la primera. Claro está, condicionando el resultado a que las características de la población y la dirección del centro fueran semejantes en las restantes. Lo que mueve esta estrategia es el intento de comprender el fenómeno en profundidad, encontrar claves que nos permitan reconocer lo hallado en otras situaciones semejantes.

Cerca de esta concepción estratégica está el diseño de investigación a través de estudios de caso (Yin 2009). La estrategia que plantea este tipo de estudios se basa en investigar todas las facetas de un caso concreto que nos sirve de referencia comprensiva del tema estudiado. En nuestro campo, la clave está en que el caso se pueda concretar en un solo centro. Si esto sucede, la mejor estrategia es plantear la investigación como si de una observación participante se tratara. Seguro que necesitaremos utilizar varias técnicas y fuentes de información. Seguro que necesitaremos acceder en varias ocasiones a los sujetos de estudios y, por tanto, al centro.

Por el contrario, si lo que buscamos es la representatividad estadística o sociológica, la elección de varios centros es obligada. La diversidad de culturas, las siempre existentes particularidades de los centros, las características poblacionales y la amplitud del universo nos conduce a esta decisión estratégica. El conocimiento de las características de la población y de los centros respecto de nuestro objeto de estudio es clave para seleccionar la muestra, mediante la identificación de estratos.

Si nuestra opción es la realización de una encuesta hay que tener mucha precaución con la forma en que se construye el cuestionario y el modo en que se va a aplicar. Ante todo, hay que considerar el alejamiento normativo de referencia de gran parte de la población penitenciaria y sus dificultades de lectura y comprensión. Lo que para el investigador puede parecer normal quizá esté muy alejado de las normas culturales de los encuestados. Por ejemplo, en nuestro último trabajo se preguntó sobre una valoración del pasado y su proyección en el futuro, pues bien, la cuestión no se entendía. Para alguien cuya preocupación se remite al corto plazo, esta temporalidad futura no se inserta entre sus referencias.

Cuestiones como escalas, preguntas de selección múltiples y formatos complejos pueden provocar el desconcierto y acabar en la no respuesta. Una redacción con un lenguaje oscuro o demasiado culto es siempre un problema en un cuestionario, como instrumento de investigación; pero puede provocar incluso el abandono de la participación en este espacio. El pre-test es obligado.

Respecto a la forma en que se aplica el cuestionario, según nuestra experiencia, nos dice que lo pertinente es que la administración sea gestionada directamente por el equipo de investigación. Cuando esta tarea se deriva a profesionales 
de la institución o a voluntarios los sesgos que se introducen y los errores pueden llegar a invalidar los resultados. Lo más grave es que se pierde el control sobre la administración. Además, el cuestionario se debe pasar de manera individual y con entrevista cara a cara. $\mathrm{O}$, al menos y si no queda más remedio, hacerlo con grupos pequeños y homogéneos según el nivel lecto-escritor. Cuando realizamos una encuesta no podemos olvidar el elevado número de analfabetos totales y analfabetos funcionales que hay en la cárcel. Si no se tiene en cuenta, este grupo se puede introducir sesgos importantes.

Si nuestra opción es la utilización de técnicas cualitativas como la entrevista o los grupos de discusión, las decisiones estratégicas se darán en los procesos de selección de los sujetos y los espacios para llevar a cabo el trabajo de campo. Los procesos de selección deben ser definidos por perfiles concretos y se debe explicar al participante el proceso de su selección. La sospecha es una cuestión que puede condicionar las respuestas e, incluso, provocar el rechazo. Los espacios más adecuados, como ya se ha mencionado, son los del área socio cultural. Los "informantes clave" son de gran ayuda. Los grupos son muy complejos de guiar. La mejor técnica es la entrevista abierta.

\section{SALIENDO DE LA CÁRCEL}

El objeto de este trabajo era doble. Por un lado, mostrar los caminos materiales de procedimientos básicos, para poder llevar a cabo investigaciones en las cárceles. Así, se ha ofrecido el contexto orgánico de las diversas concreciones de centros que albergan penados, durante el tiempo que están cumpliendo una sanción penal de privación de libertad. Cada tipo de centro comporta unas diferenciales características que estrechan o amplían el margen de opciones para la observación empírica sociológica.

Por otro lado, apuntar oportunidades y limitaciones que plantea este espacio para la producción de evidencias empíricas aceptables por el sistema de la ciencia. Apunte que se ha centrado especialmente en la observación en la cárcel para la cárcel, siendo su potencial proyección práctica principal las políticas penitenciarias en particular o la política criminal en general. Ha quedado pendiente la proyección de los condicionantes que experimentan los procedimientos de producción de evidencia sociológica desde las cárceles al exterior. A lo sumo, cuando ha sido pertinente, se ha propuesto que, más que una oposición dentro/ fuera de la cárcel, hay un marco común a ellos, que toma en su aplicación en el espacio carcelario concreciones específicas, despertando aún más la conciencia metodológica.

Por último y a modo de resumen, se ofrece el siguiente cuadro (Cuadro 4) que tiene su origen en la división inicial entre investigación criminológica e investigación sociológica de prisiones. Una división en muchos casos más formal que sustancial; pero que aquí se toma con objetivos didácticos, resaltando el acento que pone cada una de las perspectivas. No quiere decir que lo que interesa a la investigación sociológica no interesa a la investigación criminológica; ni, a 
la inversa, que lo que principalmente interesa a la perspectiva que hemos catalogado de sociológica en las prisiones, no interese a la perspectiva criminológica. A modo de conclusión, el cuadro intenta sintetizar parte de lo desarrollado en este trabajo.

En la perspectiva criminológica, se pone el acento en la identificación y explicación de procesos criminógenos. El centro penitenciario es el lugar en el que indagar sobre los comportamientos que han llevado hasta allí. Sus objetivos diferenciados tienden a ser: el registro de trayectorias delictivas, con una especial relevancia a la biografía de los sujetos; conocimiento de los contextos sociales y de las potenciales culturas criminógenas en el que ha tenido lugar la vida de los sujetos; o los procesos de socialización, cambio y culturas específicas de las que pueden considerarse profesiones delictivas, en cuanto se constituyen en formas materiales de vida de los sujetos. Desde la perspectiva que hemos denominado de la investigación sociológica de la prisión, se pone más el acento en la institución, en lo que la institución hace, partiendo la diferencia -especialmente desde el análisis funcional- entre lo que la institución dice que hace -sus objetivos manifiestos- y lo que hace, con respecto al sistema social. Sus objetivos específicos pueden ser tan variados como en el caso de la investigación sociológica: evaluación algún tipo de actuación específica realizada en el entorno y bajo la tutela de la prisión, como pueden ser cada uno de los variados programas de rehabilitación; conocer el denso contenido de la vida en prisión (procesos de socialización/resocialización, integración/exclusión, uso de los espacios y los tiempos, etc.); evaluación de un servicio público como es el ofrecido por cada uno de los centros penitenciarios, una evaluación que, a su vez, puede tener múltiples perspectivas (costes, eficiencia, resultados, impacto, etc.); relación entre los centros penitenciarios y sus respectivos entornos sociales; conocer los dispositivos y comportamientos de resistencia desarrollados por los sujetos en estas instituciones totales, etc.

Los distintos objetivos conducen a dar preferencia al uso de unas prácticas de observación empírica sobre otras. Así, teniendo en cuenta los objetivos propuestos en la perspectiva criminológica, son más pertinentes prácticas de investigación centrados en la reconstrucción de la trayectoria vital de los sujetos: historias de vida, entrevistas con un importante componente narrativo, relatos de vida, autoinformes. En el caso de la perspectiva sociológica en prisiones, para la evaluación de los denominados efectos, parecen pertinentes diseños de tipo experimental. Para la evaluación del servicio público prisión y tal como se hace en la evaluación de otros servicios públicos, la gama de prácticas de investigación es más abierta, aun cuando hay que reconocer el papel central que ocupa en ellos la encuesta con cuestionario estandarizado. Para los otros objetivos señalados en este enfoque, la entrevista en particular y las prácticas desde la perspectiva cualitativa en general parecen más adecuados.

En cuanto a los informantes, no cabe duda de que, en una y otra perspectiva, los internos ocupan un lugar central. Tal vez, el mayor horizonte macrosocial que se ha dibujado en la perspectiva sociológica de la prisión la hace más abierta a la participación del resto de actores presentes en la institución: gestores polí- 
ticos, gestores técnicos, profesionales, funcionarios, familiares de los internos, vecinos de los centros penitenciarios, etc.

Siendo el interno el informante principal, en una y otra perspectiva tiende a ser concebido de forma relativamente distinta. En la perspectiva criminológica, en su búsqueda de identificar las causas sociales del delito, hace hincapié en la concepción del interno como el producto de un proceso social, de la sociedad en la que ha desarrollado su existencia, hasta su llegada a prisión. En la perspectiva sociológica de la prisión, se pone más el foco en lo que hace la institución con el interno y, por lo tanto, a concebirlo como el resultado de la propia institución.

Por último y con la finalidad de romper con la dualidad aquí utilizada, resaltar que ambas perspectivas comparten ventajas y problemas metodológicos. Entre las primeras, contar con informantes que, en mayor o menor grado, están disponibles para colaborar, de los que se tienen registros importantes -más allá de los que ellos mismos puedan informar- y que tienen tiempo para dar a la observación empírica. Entre los problemas, a los que se ha dado especial relevancia en este trabajo, destacan aquellos relacionados en primer lugar con la representatividad: hay que tener en cuenta que sólo se observa a los que han sido "pillados", cuando es posible que otras categorías sociales cometan los mismos u otros delitos, sin que pasen por prisión; entre otras cosas porque hay categorías sociales que están menos vigiladas -habitualmente pertenecen a las categorías sociales o sectores sociales de los vigilantes- $\mathrm{y}$, en el caso de que sean detenidos, tienen menos probabilidad de ingresar en prisión. Es decir, desde el punto de vista de la representatividad de la comisión de delitos, para ver cómo funciona el proceso social de su génesis, nos encontramos con el problema de que no son todos los que cometen delito los que están (en prisión). Al menos, desde una concepción probabilística. De esa manera podría llegarse a la conclusión de que se tienen como contextos sociales criminógenos aquellos contextos que son más vigilados, por considerarse previamente como criminógenos.

Relacionado con la representatividad y tal como ha sido apuntado en el artículo, está la selección que pueda hacerse de los informantes por el propio centro penitenciario. Aquí el investigador ha de estar atento al control de tales procesos, aun cuando ha de reconocerse la dificultad para que sea el propio investigador el que lleve a cabo la selección.

Especialmente centrados en la perspectiva de la investigación sociológica en prisión, se encuentran los problemas derivados de la alta reactividad mediante. El interés en la participación por parte de los informantes en el proceso de observación tiende a ir bastante más allá de la mera contribución al conocimiento, pudiendo existir expectativas de recompensas más o menos inmediatas por el hecho de tal participación. Esto puede condicionar enormemente las informaciones que se recojan durante el trabajo de campo.

En la medida que se estudie el resultado de la prisión como institución evaluadora, la investigación puede quedar supeditada a los indicadores de rehabilitación que use la propia institución, que suelen estar bastante restringidos a los procedimientos de calificación que se llevan a cabo en la misma. Llevar el análisis de la efectividad de la prisión más allá de sí misma es posible; pero 
requiere procedimientos de observación específicos, donde dimensiones como la reincidencia o la profundización cualitativa en las trayectorias penitenciarias cobran especial relevancia.

Por último, intentando integrar buena parte de los problemas ya insertos en la reactividad, hay que tener en cuenta la distancia entre la cultura de la observación y los observadores, por un lado, y la cultura de los observados. Algo que va más allá, aun siendo esto muy importante, de la diferencia en capital formativo o incluso de idioma. Tal vez se use el mismo idioma, hasta las mismas palabras, pero insertas en sistemas culturales tan distintos, que pueden tener sentidos muy diferentes. 


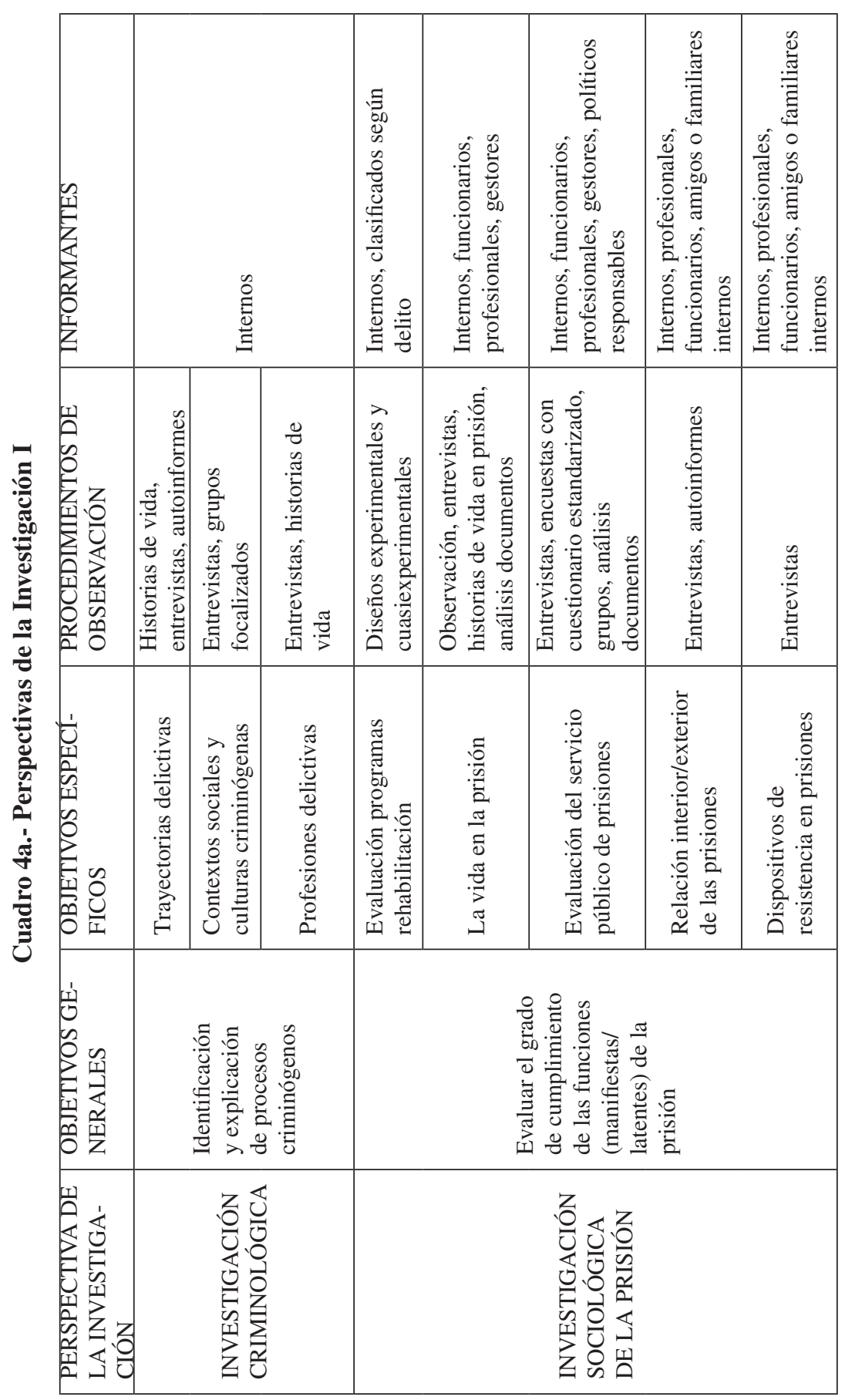

EMPIRIA. Revista de Metodología de Ciencias Sociales. N. ${ }^{\circ} 42$ enero-abril, 2019, pp. 165-195. ISSN: 1139-5737, DOI/empiria.42.2019.23256 


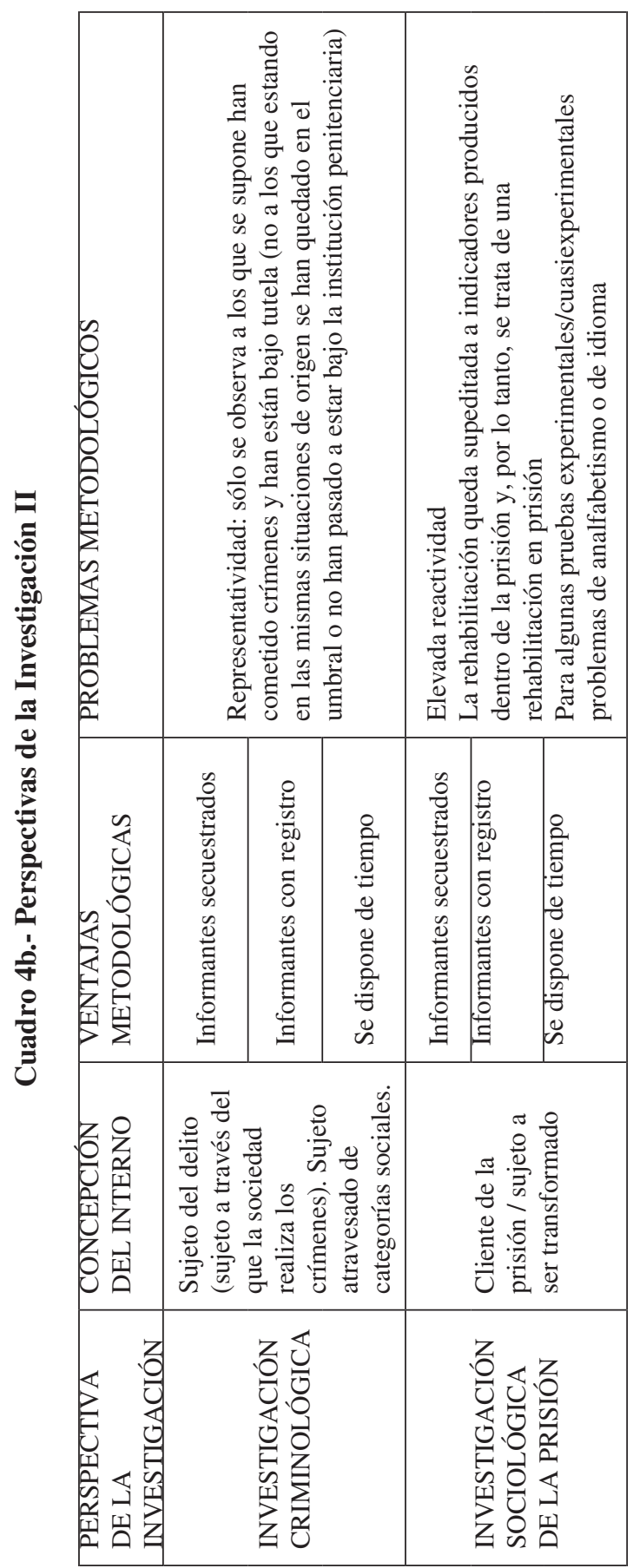

EMPIRIA. Revista de Metodología de Ciencias Sociales. N. 42 enero-abril, 2019, pp. 165-195. ISSN: 1139-5737, DOI/empiria.42.2019.23256 


\section{BIBLIOGRAFÍA}

Almeda, E. (2002). Corregir y castigar: el ayer y hoy de las cárceles de mujeres. Bellaterra, Barcelona.

Almeda, E. (2017) "Criminologías feministas, investigación y cárceles de mujeres en España”. Papers. Revista de sociología. Vol. 102, № 2, págs. 151-181, 2017. (Ejemplar dedicado a: Mujeres, delitos y prisiones),

Basaglia, F. y Ongaro, F. (2010) La maggioranza deviante. L'ideologia del controllo sociale totale. Milán. Dalai Editore.

Becker, H. (1963) Outsider. Studies in The sociology of Desviance. Nueva York. [Hay traducción en castellano. Outsiders. Hacia un sociología de la desviación. Siglo XXI 2009]

Callejo, J. y Viedma, A. (2005) Proyectos y estrategias de investigación: la perspectiva de la intervención. Madrid, Mac Graw Hill.

Carlen, P. (1998) Sledgehammer: Women's Imprisonment at the Millennium. London: Macmilla.

Cervelló, V. (2016) Derecho penitenciario. Tirant Lo Blanch. España.

Cood H. (2008) In the Shadow of Prison. Families, imprisonment and criminal justice. Routledge, Nueva York.

Cohen S. (1972) Folk Devils and Moral Panics. Macgibbon \& Kee. Londres. [Hay traducción en castellano. Demoinos populares y pánicos morales. Gedisa, Barcelona 2017]

Cohen, S. y Taylor, L. (1972) Psychological survival: The experience of Long-term Imprisonment. Harmondswordth: Penguin Books.

Coller, X. (2000) Estudio de casos. Madrid: Centro de Investigaciones Sociológicas.

Crew, B. y Bennett, J. (2012) The Prisonner. Routledge, Londres y Nueva York.

Del Val, C. y Viedma, A. Coord. (2012) Condenadas a la desigualdad. Icaria.

Fernández, L. y Nistal, J (2012) Manual de Derecho Penitenciario. Thomson Reuters, Aranzadi.

Foucault, M. (1975). Surveiller et punir naissance de la prison. Gallimard. París.

[Hay varias traducciones en español. Vigilar y castigar. El nacimiento de la prisión (2000) Madrid. Siglo XXI]

Goffman, E. (1972) Internados: ensayos sobre la situación de los enfermos mentales. Buenos Aires, editorial Amorrortu [e.o 1961].

Haney, C., Banks, c. y Zimbardo P.G (1973) "Interpersonal dynamics in a simulated prison”, Interntaional Journal of Cryminology and Penology, 1, págs. 69-97.

Ibáñez, J. (1979) Más allá de la sociología. Madrid. Siglo XXI.

Jewkes, Y. (2014) "Special Section Editorial” Qualitative Inquiry. SAGE. Londres.

Laub, J.H. y Sampson, R. J (2006) Shared Beginnings, Divergente lives. Delinquent boys to age 70 . Harvar University Press. Cambridge.

Liebling, A. (1999) "Doing research in prison. Breaking the silence?" en Theoretical Criminology. Vol. 3(2): 147-173; 008078.

Liebling, A. y Maruna, S. Edt. (2005) The effects of Imprisonment. Willan Publishing, Portland.

Luhmann, N. (1996) La Ciencia de la Sociedad. México. Universidad IberoamericanaAnthropos.

Marcuse, H. (2010) El hombre unidimensional. Barcelona. Ariel [e.o. 1964].

Maura, S. y Immarigeon, R. (2005) After Crimen and Punisment. Willam Publishing. Portland. 
Ríos Martin, J. C y Cabrera, P.J. (2002) Mirando al abismo. El régimen cerrado. Universidad Pontificia de Comillas. España.

Ríos Martín y otros (2018). Manual de ejecución penitenciaria. Defenderse de la cárcel. Universidad Pontificia de Comillas. España

Rosa, H. y Scheuerman, W.E. (2009) "Introduction", en H. Rosa y W. E. Scheuerman (eds.) High-Speed Society. Pensilvania. Penn State University.

Sampson, R. J. y Laub, J. (1993). Crime in the making. Pathways and turning points through life. Harvard University Press.

Saw, C.R. y Burguer E. W. (1966) The Jack Roller: A Delinquent Boy $\bigotimes_{s}$ Own Story . University Chicago Press. (e.o 1930)

Scarce, R. (2002) "Doing time as an act of survival" Symbolic Interaction, Volume 25, Number 3, pages 303-321, ISSN 0195-6086; online ISSN 1533-8665.

Sutherland, D. (1956) The Professional Thief Chicago. [Hay traducción en castellano: Ladrones profesionales. La piqueta, Madrid 1998]

Sykes, M. G (2007). The Society of Captives: a study of maximum security prison. Princeton: Princeton University Press [e.o 1958] [Hay edición en español publicada en 2017 por Siglo Veintiuno editores].

Viedma, A. (2013) "Tiempo de estudio vs tiempo de prisión. Identidad y resistencia frente al tiempo de castigo". Actas Congreso XII Federación Española de Sociología.

Wahidin, A y Moore, L. (2011) "Ethics and criminological research" en Davis, P. Francis, P y Jupp, V. Doing Criminological Research. SAGE, Londres.

Whyte, W. F. (1993) Street Corner Society: The Social Structure of an Italian Slum. University Press Chicago. [e.o 1943] [Hay traducción en castellano: La sociedad de la esquina. Centro de Investiaciones Sociológicas, 2015]

Yin, R. K. (2009) Case Study Research. Design and Methods. SAGE. 
